\title{
A Prospective Randomised Control Trial for Comparison Between olecranon Lock Plate And Tension Band Wiring In Transverse olecranon Fractures
}

\author{
P.Sarma ${ }^{1}$,B.Agarwal ${ }^{2}$, K.Hazarika $^{3}$, P.Agarwal ${ }^{4}$, A.Gaikwad $^{5}$ \\ Gauhati Medical College, Guwahati,Assam
}

\begin{abstract}
Introduction: Olecranon fractures are frequently encountered in the practice of orthopaedic surgery with a prevalence of $60 \%$ among patients with a fracture of the proximal ulna. Generally the treatment of choice for displaced olecranon fracture is open reduction and internal fixation. We report the result of a prospective Randomised Control Trial of Forty patients, who were treated either by Tension Band Wiring or Olecranon Lock Plate.

Methodology: In a prospective controlled study, 20 cases each of transverse olecranon fractures were treated with Tension Band Wiring and Olecranon Lock Plate respectively. The age group of the patients is 18 to 70 years, The surgery and follow-up conducted at the same centre. Patients will be followed up at regular intervals and outcome variables assessed and recorded

Results: In our study overall satisfactory result (excellent+ good) according to Mayo Elbow Performance Score was obtained in $92.5 \%$ cases. In the TBW group satisfactory result were obtained in $95 \%$ cases whereas in Olecranon Lock Plate group it was obtained in $90 \%$ cases.

Conclusion: This prospective study presents the technique applied in the treatment of transverse olecranon fractures. The results obtained in this study have shown that the both Tension band wiring and Olecranon Lock Plate techniques are safe, convenient and effective, since there was no obvious damage, nor major complications.
\end{abstract}

Keywords: Olecranon, Tension Band Wiring, Lock Plate

\section{Introduction}

The fracture of the olecranon is an intra-articular injury with loss in the extension mechanism of the elbow joint, therefore its treatment is always operative. The main objective of operation is to achieve adequate reduction and rigid fixation, allowing early mobilization in an attempt to reduce morbidity due to stiffness. Weber and Vasey in 1963 introduced tension band wiring for its treatment but several modification attempts were undertaken, especially to avoid migration of the Kirschner wire. Fractures can be approached by an open reduction and fixation using lag screws, plates, interosseous wires.

The common causes of Olecranon fractures are mostly due to self fall ,motor vehicle crash, fall from a height, or a direct blow to the elbow and are commonly observed orthopedic injuries in the emergency room. Different classification methods are present to asses the fracture patterns, among which Mayo's classification system is considered to be the most commonly used.They depend on the degree of fracture displacement, the stability of the elbow joint and comminution at the fracture site. Undisplaced fractures are Mayo Type I fractures and these are exceptional and can be managed conservatively. However, most olecranon fractures are displaced, considered as Mayo Type II and Type III, and in such cases operative treatment is recommended. The primary objective of operative treatment of olecranon fractures is anatomic restoration of the trochlear notch. Displaced olecranon fractures can be treated with a tension band construct for adequate stability and early mobilization. Plate and screw fixation may ensure stronger fixation in the case of comminution or a fracture-dislocation which tension band wiring cannot.

\section{Materials And Methods}

A prospective randomised controlled trial study was carried out for a total of 40 cases of transverse olecranon fractures aged between 18yrs - 70yrs attending the OPD and Emergency department of Orthopaedics, Gauhati Medical College \& Hospital from May 2014 to may 2015 and followed up for a minimum of 12 months. All transverse olecranon fractures < 3weeks old in patients aged 18-70yrs of both sexes with Intact neurological and vascular status and No associated fractures in same limb were included All the patients in this study were admitted either through out-patient department or through emergency department of the college. On receiving the patient, initial symptomatic treatment and a detailed history of the patient in regard to age, sex, socio-economic background, duration \& mode of injury, previous illness or treatment, was taken as per the 
proforma. A thorough clinical examination was carried out to exclude any associated injury \& other medical and surgical illness which may require necessary treatment. Injured limb examined thoroughly for open fractures, distal neurovascular status and to exclude compartment syndrome. The limb is initially immobilized with a long arm/above elbow posterior POP slab. Initial radiographs were done taking true antero-posterior and lateral views of the elbow. Patient and the family is then explained about the nature of the fracture, prognosis of non operative and operative treatment and also regarding the need for open reduction and internal fixation in the case.

The limb was kept elevated in an arm pouch. With this, gradual flexion extension exercises were started within the limit of tolerance of pain. The patients are then discharged and called for follow up and suture removal at 10- $14^{\text {th }}$ post operative day. subsequent follow up were done at $6,12,18$, 24 weeks and then 2 monthly interval

\section{Results}

40 adult (Aged 18to 70 years) patients with transverse olecranon fractures were chosen for the study and randomly allocated into two of the following treatment groups according to the randomization table

1. Open reduction and internal fixation with tension band wiring (TBW) $(n=20)$

2. Open reduction and internal fixation with Olecranon Lock Plate $(n=20)$

All the patients were followed up for a minimum period of 12 months with average follow up of 14 months. Follow up was carried out at 2, 6, 12, 18, 24 weeks and then at 2 monthly intervals. Full assessment was done at the end of 12 months from the date operation in all cases. In our study, Average Flexion in TBW group is $120^{\circ} \&$ in OLP group is $116^{0}$ Van Der Linden found the average flexion to be $133^{0}+/-11^{0}$. In our study, Average loss of extension in TBW group is $14.5^{\circ} \&$ in OLP group is $15.75^{\circ}$ Karlson found to be $15^{\circ}+/-17^{0}$ in his study In our study, Average supination and pronation arc in TBW group is $111.25^{\circ}$ and in OLP group is $113.75^{\circ}$ Lindenhovius found Average supination and pronation arc to be $81^{\circ}+/-15^{\circ}$ and $70^{\circ}+/-30^{\circ}$ in his study. The functional results were evaluated according to Mayo Elbow Performance scoring system (MEPS). With a maximum possible score of 100, the results are graded as excellent for scores >90; good for scores 75-89; fair for 60-74 and poor for scores less than 60. At the end of six month we evaluated our results for each patient in terms of total Mayo elbow performance score and graded them accordingly. Overall we obtained "excellent" results in $30 \%$; "good" in $62.5 \%$ cases; "fair" in $5 \%$ and "poor" in $2.5 \%$ cases with mean MEPS of 83. Therefore a satisfactory result was obtained in $72.5 \%$ cases.

Chalidis et al 2008, raised the question if TBW is still the "gold standard" in the treatment of olecranon fractures and achieved $85.5 \%$ satisfactory result after treatment. Calculating the results in the implant groups in our study, we obtained "excellent" results in 35\%; "good" in $60 \%$ cases; and "poor" in $5 \%$ cases with a mean MEPS of 84.75 in the tension band wiring group (TBW) whereas in OLP group we found "excellent" results in 35\%; "good" in 55\% cases; and "fair" in 10\% cases with a mean MEPS of 81.5 . Therefore satisfactory results obtained in $95 \%$ and $90 \%$ cases for the tension band wiring group (TBW) and OLP group respectively. This finding is correlating and is consistent with the above mentioned studies with respect to the type of wiring used and over all outcomes. On statistical analysis comparing the two groups, the p- value (0.462) was found to be which is statistically insignificant. The criteria of Anderson et al (1975) were taken into account to assess the union of the fracture. A fracture was defined as healed when there was obliteration of fracture line and evidence of bridging trabeculae. Accordingly, all of the fractures in both the study groups united without the need for a second procedure, before 6 months with an exception in TBW, where non-union occurred. Thus the union rate was $97.5 \%$ in both the groups in the study.

The time taken for union in the TBW group ranged from 6 to 13 weeks with a mean of 10 weeks. The time taken in the OLP group for radiological union ranged from 8 to 14 weeks, with a mean of 10.6 weeks. The two groups were tested using the unpaired t test to bring about the presence of a significant difference, if any between the means of time for union in the two groups. The p value was 0.39 which is considered not significant and shows that time for union does not depend on the type of implant used.

Hume and Wiss in 1992 and Fan et al. in 1993 reported that union rate in their studies was 14 weeks.

According to Wolfgang G et al. 1987, tension-band wiring usually provides stable fixation with a high union rate for simple non-comminuted transverse olecranon fractures.

Papagelopoulos and Morrey, 1994 reported nonunion of olecranon fractures up to $1 \%$ of patients, with typical symptoms of pain, instability, or loss of motion. 2 cases of superficial infection were found in TBW group and 1 in OLP group. Hardware prominenence was found in 7 cases of OLP group and in 3 cases of TBW group. However 1 case of non union was found in TBW group which was re operated with OLP and bone grafting and united after 16 weeks of second surgery. no such non union was noted in OLP group 


\section{Conclusion}

This prospective study presents the technique applied in the treatment of transverse olecranon fractures. Choice regarding the type of implant has always been a controversial issue.In our study, we have seen that there is a little difference between Tension band wiring and screw fixation in terms of final functional outcome. The results obtained in this study have shown that the both Tension band wiring and Olecranon lock plate techniques are safe, convenient and effective, since there was no obvious damage, nor major complications. Hardware prominence was a major problem in OLP group In addition, most of the fractures healed within a similar healing time to other methods with both good alignment and function of elbow joints. But there is clear indication for a detailed prospective randomized controlled study, involving a significant larger number of subjects for a longer duration, to prove the further clinical utility of these techniques

\section{References}

[1]. Rommens PM, Schneider RU, Reuter M. Functional results after operative treatment of olecranon fractures. Acta Chir Belg. 2004;104(2):191-7.

[2]. Matthews LS, Green CA, Goldstein SA. The thermal effects of skeletal fixation-pin insertion in bone. J Bone Joint Surg [Am] 1984;66:1077-1083.

[3]. Court-Brown CM, Koval KJ. The Epidemiology of Fractures. In: Bucholz RW, Heckman JD, Court- Brown C, editors. Rockwood and Green's Fractures in Adults. 6th ed. Lippincott, Williams \& Wilkins; 2005. p.95-144.

[4]. Pun WK, Chow SP, So YC, Luk KD, Ip FK, Chan KC, Ngai WK, Crosby C, Ng C. A prospective study on 284 digital fractures of the hand. J Hand Surg [Am]1989;14:474-481.

[5]. Iannuzzi N, Dahners L. Excision and advancement in the treatment of comminuted olecranon fractures. J Orthop Trauma.2009;23(3):226-8.

[6]. Black D, Mann RJ, Constine R, Daniels AU. Comparison of internal fixation techniques in metacarpal fractures. J Hand Surg [Am] 1985;10:466-472.

[7]. Villanueva P, Osorio F, Commessatti M, Sanchez-Sotelo J. Tension-band wiring for olecranon fractures: analysis of risk factors for failure. Journal of shoulder and elbow surgery/ American Shoulder and Elbow Surgeons. 2006;15:351-356.

[8]. Morrey BF. Current concepts in the treatment of fractures of the radial head, the olecranon and the coronoid. Journal of Bone and Joint Surgery. 1995;77A:316-327.

[9]. Doornberg JN, van Duijn J, Ring D. Coronoid fracture height in terrible-triad injuries. J Hand Surg [Am] 2006 May-Jun;31(5):7947.

[10]. Doornberg J, Ring D, Jupiter JB. Effective treatment of fracture-dislocations of the olecranon requires a stable trochlear notch. Clin Orthop Relat Res 2004 Dec;(429)(429):292-300.

[11]. Ring D, Jupiter JB, Sanders RW, Mast J, Simpson NS. Transolecranon fracture-dislocation of the elbow. J Orthop Trauma 1997 Nov; 11(8):545-50.

[12]. Jupiter JB, Leibovic SJ, Ribbans W, Wilk RM. The posterior Monteggia lesion. J Orthop Trauma 1991;5(4):395-402.

[13]. Villanueva P, Osorio F, Commessatti M, Sanchez-Sotelo J. Tension-band wiring for olecranon fractures: analysis of risk factors for failure. J Shoulder Elbow Surg 2006 May-Jun;15(3):351-6.

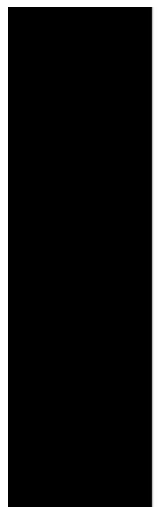

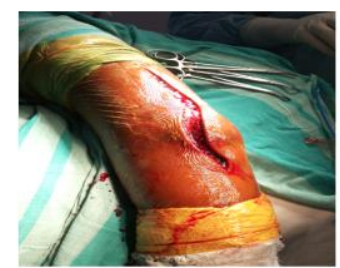

Skin incision

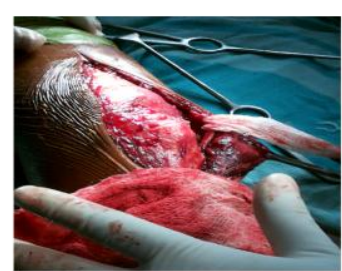

Isolation of ulnar nerve
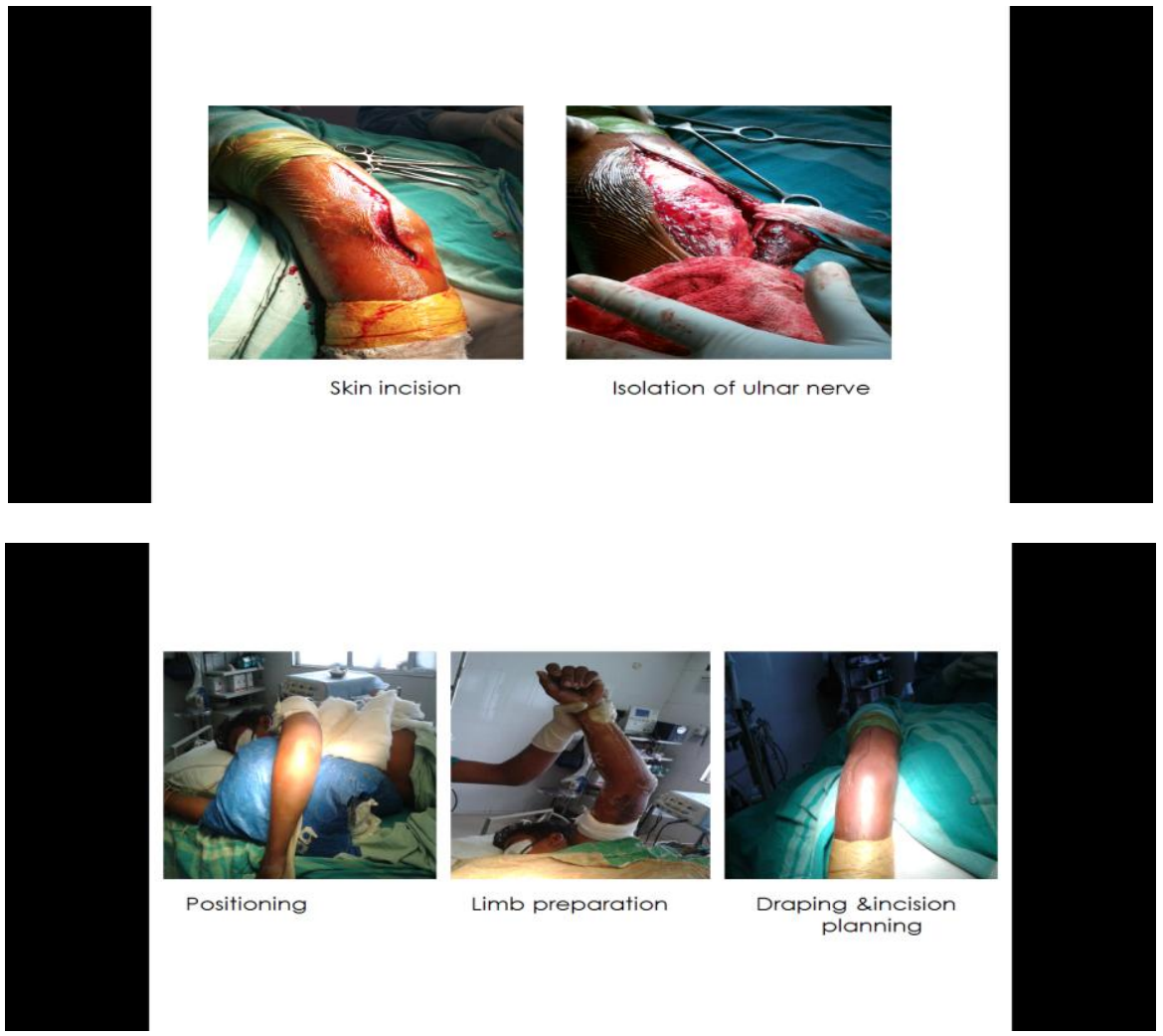

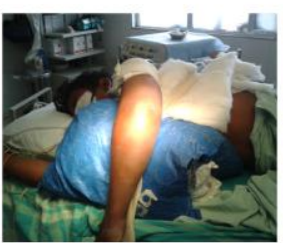

Positioning

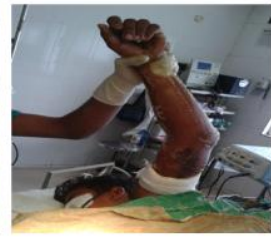

Limb preparation

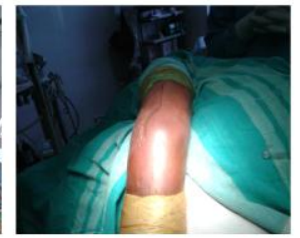

Draping \&incision planning

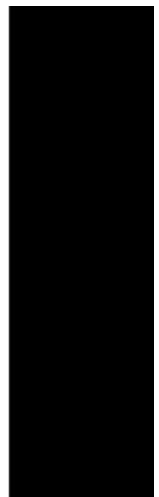



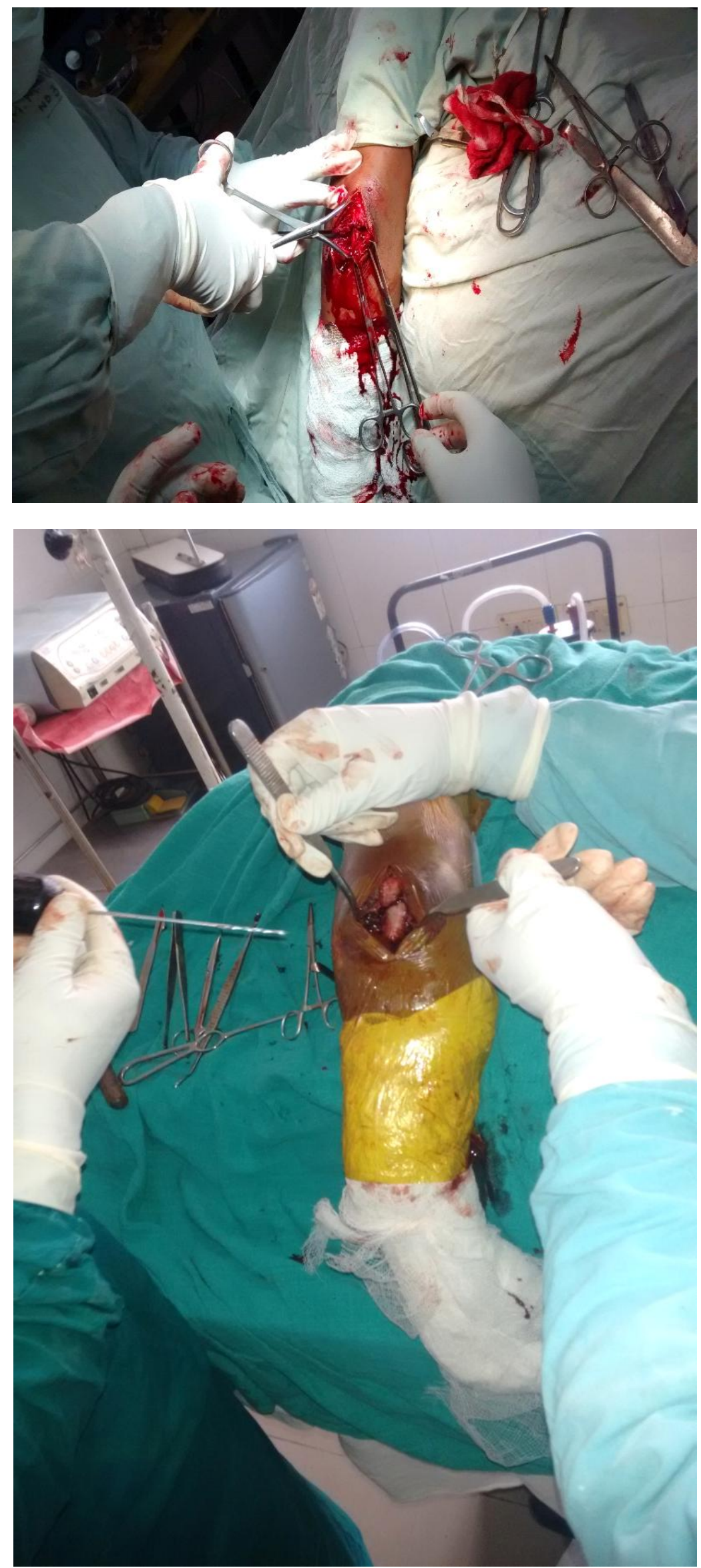

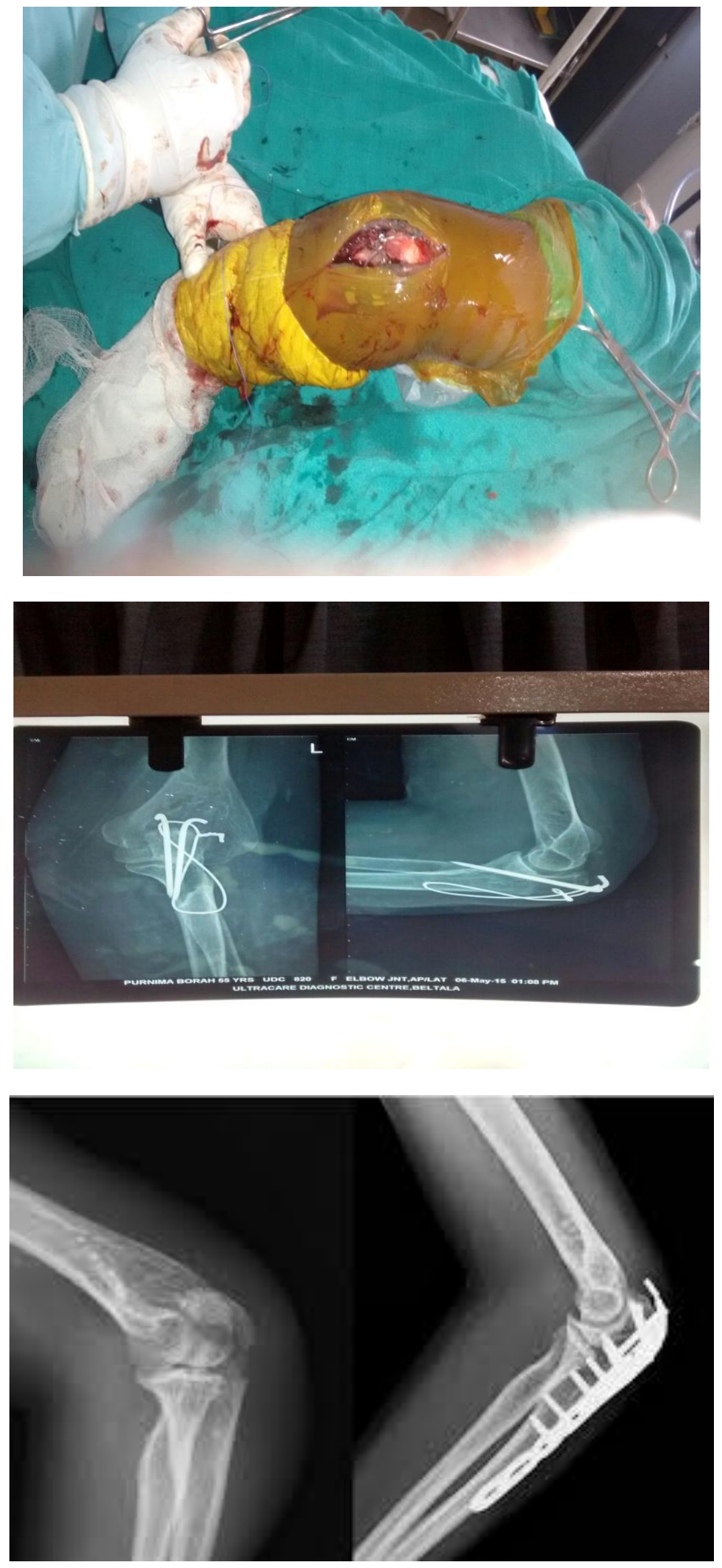